\title{
Bioelectrical activity of the amygdala of rats under conditions of chronic alcoholism and imbalance of nitric oxide
}

\author{
Olena Severynovska ${ }^{1}$, Viktoriia Babicheva ${ }^{1}$, Alexey Galinskiiy ${ }^{1,2}$, \\ Anatoliy Rudenko ${ }^{2}$, Olga Bogatyreva ${ }^{3}$, Matthew Boyko ${ }^{4}$ \\ ${ }^{1}$ Oles Honchar Dnipropetrovsk National University, Ukraine
}

${ }^{2}$ Dnipropetrovsk Gastroenterology Institute Ukrainian Academy of Medical Sciences, Ukraine

\author{
${ }^{3}$ University of Bath, BioTRIZ Ltd, UK \\ ${ }^{4}$ Soroka University Medical Center, Israel \\ E-mail address: dekanat.bef.dnu@i.ua
}

Keywords: alcohol, NO, amygdala, sodium nitroprusside, nitroarginine, aminoguanidine

\begin{abstract}
Evaluation of biopotentials in the region of the amygdala of rats in the combined influence of alcohol and drugs, causing an imbalance of nitric oxide is described in this paper. Chronic alcoholism leads to structural morphological changes in the liver. A study of the amplitude of electrical activity showed that the electrical potential in the amygdala after 8 weeks chronic alcoholism was lower compared with normal rats. The frequency spectrum analysis showed decreased in the 2 times or more absolute spectral powers of all components. The relative spectral powers of its components: $\delta: \theta: \alpha: \beta$ was as a $2: 2: 2: 4$.

The use of sodium nitroprusside (donator of NO) together with the chronic consumption of ethanol reduces the effect of ethanol on low and high-frequence portion the performance of the bioelectrical activities spectrum in the amygdala. The use of non-selective blocker of NO-synthase - nitroarginine aggravated by the braking action of alcohol. The important role of nitric oxide in the development of adaptive-compensatory reactions of the damaged body is described. Our results may be useful to assess the contribution of NO in operation amygdala, conditions of chronic pathologies, and treatment of neuropsychiatric conditions, including diseases caused by alcohol abuse.
\end{abstract}

\section{INTRODUCTION}

The amygdala is anatomically complex and heterogeneous structure consisting of more than 10 basal ganglia of the brain. The amygdala forms part of the limbic system. The amygdala is a brain structure that is essential for decoding emotions and in particular stimuli that are threatening to the organism [2].

According to American scientists from Duke University if MRI research revealed an imbalance in the activity of ventral striatum and the amygdala, one can likely expect the development of alcohol dependence and heightened sexuality [7].

Unfortunately, the problem of alcoholism is very common in many countries around the world.

The brain tissue easy saturates ethanol and its concentration is higher than in other organs due to intensive blood supply [1]. Using the radiographic method it was shown that the ethanol concentration in the gray matter higher than in white [3]. According to our research a central nucleus of the amygdala (AMG) complex is the most significant area where we can expect the most influence of alcohol because it consists of almost all the characteristic of the complex cell types. It is situated between the nuclei of the lateral and medial parts of the AMG [9]

Numerous experiments with injury and stimulation amygdala show that the involvement in the formation of endocrine and visceral components of the various forms of species-specific behavior [4, 14]. Also there is a large amount of evidence of the important role of nitric oxide (NO) in the processes of the central regulation of visceral functions [6]. Population of NO-synthesizing 
neurons was found in the AMG complex. We can assume that one of the mechanisms of implementation of AMG influences on the structure of the autonomic neuron network is a change of NO-synthesizing activity of neurons in the amygdala. It can be as a result of changes in the level of excitability of cells innervated by AMG structures, and a consequence of changes in their relations with other brain structures, including the nuclear of the amygdala complex. However, there is no experimental evidence supporting this conclusion.

Meanwhile, the study of involvement of NO in the modulation of electrical activity of the amygdala (as one of the most important centers of limbic system), is of interest not only for fundamental research but also for clinical practice. Special studies have shown that AMG may be involved in the process of developing a number of neurological and psychosomatic disorders [5] and NO -dependent mechanisms play an important role in this [6]

\section{MATERIALS AND METHODS}

\section{Animals}

Male Wistar rats weighing about 250-300g, were purchased and maintained under standard experimental conditions and fed with standard diet of the vivarium. All the experiments comply with the recommendations and guidelines of the Committee for the Purpose of Control and Supervision of Experiments on Animals, Ukraine.

Selection of experimental groups of animals was done according to their individual and psycho-emotional characteristics. Behavioral changes were analyzed in the open-field and supported by plus-maze tests and the test for resistance to hypobaric hypoxia, which made it possible to select animals with similar reactions [10].

The experiments were conducted on 60 rats divided into five groups:

I group - intact (control) males of Wistar rats; II group - males of Wistar rats were subjected to an oral ethanol self-administration procedure with a forced diet where they were offered $10 \%$ $(v / v)$ ethanol solution for 60 days and 10 days of intraperitoneal introduction of $16 \%$ ethanol at $4 \mathrm{~g} /$ $\mathrm{kg}$; III group - males of Wistar rats were subjected to an introduction of the donor nitric oxide sodium nitroprusside in a dose of $2.5 \mathrm{mg} / \mathrm{kg}$ for 10 days on a background similar to alcoholism how II group; IV group - males of Wistar rats were exposed to introduction of a selective blocker of inducible NO-synthase - aminoguanidine at a dose of $50 \mathrm{mg} / \mathrm{kg}$ for 10 days on a background similar to alcoholism how II group; V group - male Wistar rats were subjected to introduction of non-selective blocker of NO-synthase - nitroarginine for 10 days on a background similar to alcoholism how II group.

The solutions were prepared just before the experiment (according to the manufacturer's recommendations preparations), and administered to test animals at the same time - 9:00.

\section{METHODS OF STUDY OF BIOELECTRIC ACTIVITY OF THE AMYGDALA}

After 18 hours of food deprivation with free access to water, the rats were anesthetized with a solution of urethane $(1.5 \mathrm{~g} / \mathrm{kg})$. Anesthetized animals were fixed in stereotactic device. Registration of bioelectric activity of the central nucleus of the amygdala in rats was performed by unipolar needle platinum electrode imposed under stereotactic atlas coordinates [8]. The ground electrode was attached to the tail, indifferent - to the ears of the rats. Registration bioelectrical activity amygdala was performed using a polygraph system computer. The recording time was 120 minutes. Verification localization tips of the electrodes were carried out on frontal sections of the brain. We conducted frequency spectrum analysis records currents from the amygdala, giving the absolute $\left(\mathrm{mkV}^{2}\right)$ and relative $(\%)$ spectral power of the fundamental $(\alpha, \beta, \delta, \theta)$ components. [11, 13]. 


\section{Histopathology of liver tissue}

Liver tissue was quickly removed after euthanasia and fixed in $10 \%$ buffered formalin for 48 hours, cleaned in xylene, embedded in paraffin and sectioned (5-6 $\mu \mathrm{m}$ thickness) using a microtome. Sections were stained with hematoxylin and eosin dye.

\section{Statistical analysis}

Data were analyzed using programs MATLAB 8.4.0 and Microsoft Excel 2013. Analysis of variance (ANOVA) was used. The limit of statistical significance was set at $p \leq 0.05$.

\section{RESULTS}

The extent of the impact of alcohol intoxication on the body of rats was assessed by histological sections of the liver. Histological analysis of liver determined that in the rat of group II was the development of a complex characteristic structural change. At the basis of these changes is common micro vesicular diffuse fatty degeneration (about $80 \%$ of all cells). Around the central vein was focuses of micro and macro vesicular fatty change, mostly in the third zone of the acinus in $83.3 \%$ of cases (Fig. 1A). A significant amount of liver cells were capable to eosinophilic degeneration. They have either singly or in groups in the 2 nd and 3rd zones.

A

$\mathrm{B}$

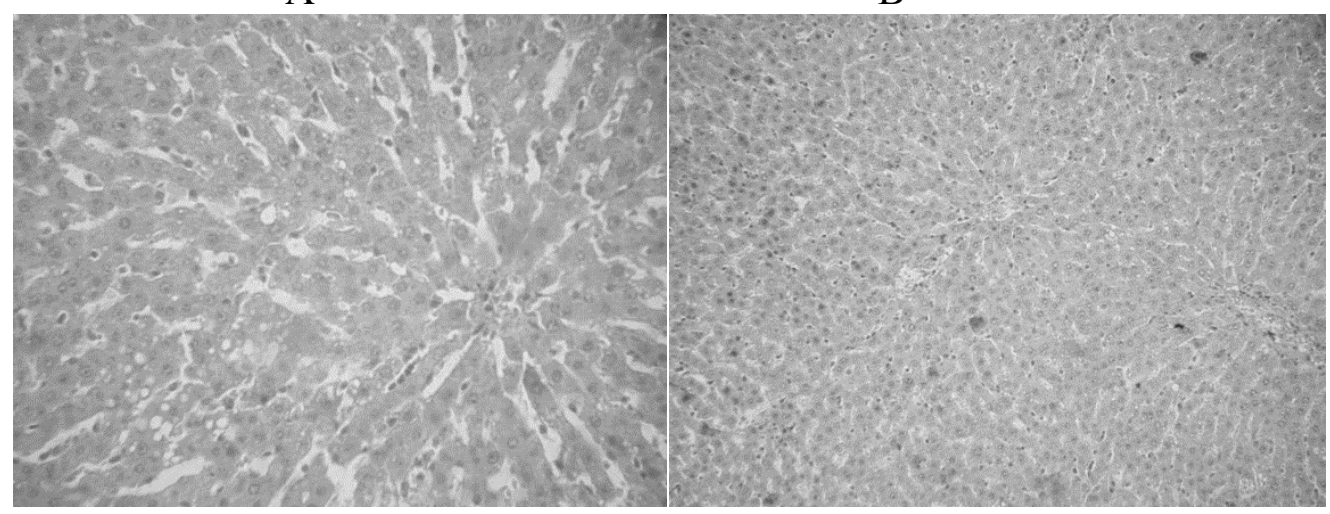

Figure 1. Photomicrographs of liver sections of the chronic alcoholism rat's (group II).

(A) Macro vesicular fatty change with mild inflammation cell with average drop steatosis.

(B) A group of hepatocytes with eosinophilic globule.

According to the literature, eosinophilic degeneration of cell, which is often accompanied by condensation of nuclear chromatin (Councilman Body), can show that these cells will soon die by apoptosis. Thus, the model experiments show a significant degree of damage to the main "biochemical laboratory" of the body as a result of alcohol intoxication.

\section{The bioelectric activity of the amygdala of control animals}

Analysis of the recording of bioelectrical activity of the amygdala of control animals allowed to mention that the summary of electrical activity of the central nucleus of the amygdala was fairly stable with a pronounced dominance of $\beta$-like activity. That is characterized of this species and applied urethane anesthesia. Relative spectral power electrical activities of amygdala characterized by the following relation its frequency components: $\delta: \theta: \alpha: \beta$, as a $2: 2: 2: 4$.

\section{The effect of chronic alcohol abuse in the bioelectric activity of the amygdala in rats}

Bioelectrical activity chronically alcoholized animals had a fairly nonstable character with predominance of $\beta$ frequency range. Absolute spectral power bioelectrical activity of amygdala was reduced in all frequency portion to $53-57 \%$ compared with results of control animals (Fig.2). Relative spectral power of bioelectrical activity of amygdala characterized by retaining a percentage of all frequency bands: $\delta: \mathrm{q}:$ a: $\beta$, as a $2: 2: 2: 4$. 

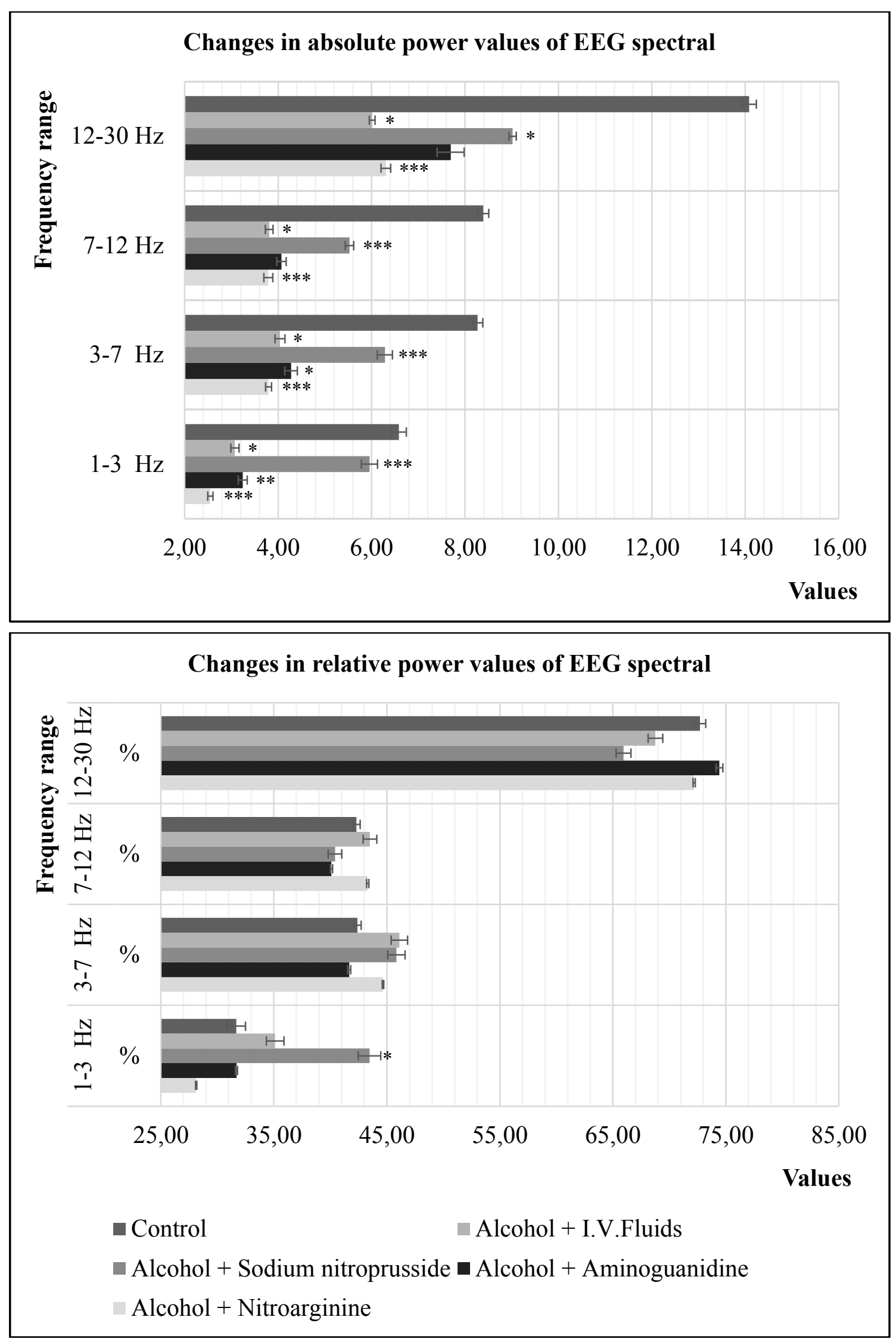

Figure 2. Value of absolute (A) and relative (B) spectral power bioelectrical activity amygdala in the control and experimental groups of animals. * - differ significantly from control $(p<0.05), * *$ differ significantly from control $(\mathrm{p}<0.001), * * *$ differ significantly from control $(\mathrm{p}<0.0001)$ 


\section{Effect of sodium nitroprusside on bioelectric activity of AMG rats with chronic alcoholism}

Although general bioelectrical activity of the amygdala of animals with chronic alcohol abuse and donator NO - sodium nitroprusside, was disorganized character, however maximum of the spectral power was in the $\beta$-fixed range. Although absolute spectral power was lower than in the control of in all frequency portion at $10-36 \%$ compared with the results of control group rats after the administration of sodium nitroprusside, but it was less visible as comparing to the animals group with of chronically alcohol. This suggests a positive effect of the donor nitric oxide in these conditions. The relative spectral power of bioelectrical activity has increased in the low-frequency and $\delta-\theta$ part and decreased in the $\alpha$ - and $\beta$-like portion.

\section{Effect of aminoguanidine on bioelectric activity of the amygdala in rats with chronic alcoholism}

Bioelectrical activity of the amygdala of animals of the IV-th group, which on the background of ethanol injected blocker nitric oxide - aminoguanidine, had disorganized character with maximum spectral power in the $\beta$-frequency psition. Records traced frequency wave activity. It expressed alternately periods of low amplitude high frequency components with bursts of $\delta$-like activity that alternated with periods of high-amplitude oscillations. Absolute spectral power in all frequency bands was reduced by $45-51 \%$ compared with the control group and did not differ significantly from that of animal alcohol. In the latter case, the exception was the dominant values in the frequency range of $12-30 \mathrm{~Hz}$. The relative spectral power was not significantly different from controls.

\section{Influence nitroarginine on bioelectric activity of the amygdala in rats with chronic alcoholism}

Recording of bioelectrical activity of AMG showed the decrease in the amplitude of the frequency components as compared with the control group as a result of the selective blocker of inducible NO-synthase amid alcoholization.

Absolute spectral power in all frequency portions in the animals of this group was decreased by $54-61 \%$ compared to the control group. It appears that the comparison with the results of II group indicates a greater influence of this blocker in the low frequency range where the power of $\delta$ and $\theta$-components mostly decreased. The results of contribution of high $\alpha$ - and $\beta$-components in bioelectrical activity of amigdala was similar to the results of animals which were under the influence of ethanol. Analysis showed the relative spectral power conservation relations of all frequency bands with the greatest decrease by $11 \%-\delta$-like activity and a slight increase in the $\theta$ and $\alpha$-like activity as compared with the control group. With this combined effect of two factors had significantly (3 times) decrease in $\delta$-like component in cmparison with the influence of alcohol.

\section{CONCLUSION}

Chronic alcoholism, causing structural morphological changes in the liver, accompanied by a decrease in the absolute spectral power bioelectrical activity amigdala rats in all frequency bands of 2 or more times. This is not disturbed the relative proportions of its components: $\delta: \theta: \alpha: \beta$, as a 2:2: 2: 4 .

The use of sodium nitroprusside (donator of nitrogen) with prolonged chronic alcoholism reduces the effect of ethanol on activity amigdaly. This was expressed in a doubled absolute spectral power bioelectrical activity the above part of the brain and their approach to the results of control. These results may indicate the important role of nitric oxide in the development of adaptive-compensatory reactions of the damaged body. This is confirmed by the worsening adverse effects of alcohol under the influence of a non-selective blocker NOS. Additional research in this area will help to understand the role of NO in the functioning of the central nucleus of the amygdala in the conditions of chronic pathologies 


\section{References}

[1] F. Campagna, S. Montagnese, S. Schiff, M. Ruzzoli, Confounders in the detection of minimal hepatic encephalopathy: a neuropsychological and quantified EEG study, Liver Int., 35(5) (2015):1524-1532.

[2] A.D. Dager, D.R. McKay, J.W Jr Kent, J.E. Curran, Shared genetic factors influence amygdala volumes and risk for alcoholism, Neuropsychopharmacology. 40(2) (2015) 412-420.

[3] R. Folescu,C.L. Zamfir, A.M. Sişu, A.G. Motoc,A.C. Ilie, M. Moise, Histopathological and imaging modifications in chronic ethanolic encephalopathy, Rom J Morphol Embryol. 55(3) (2014) 797-801.

[4] J.L. Kissler,S. Sirohi, D.J. Reis, H.T. Jansen, R.M. Quock, D.G. Smith, B.M. Walker, The onetwo punch of alcoholism: role of central amygdala dynorphins/kappa-opioid receptors, Biol Psychiatry. 75(10) (2014) 774-782.

[5] O.A Lyubashina, A.A Dorofeeva, E.B. Pluzhnichenko, S.S. Panteleev, Location of neurons in the central nucleus of the amygdaloid body projecting to the paraventricular nucleus of the hypothalamus. Neurosci Behav Physiol. 40(1) (2010) 103-105.

[6] O.A. Lyubashina, A.D. Nozdrachev, NO-dependent mechanisms of amygdalocortical influence, Dokl Biol Sci. 421 (2008) 225-228.

[7] Y.S. Nikolova, A.R. Knodt, S.R. Radtke, A.R. Hariri, Divergent responses of the amygdala and ventral striatum predict stress-related problem drinking in young adults: possible differential markers of affective and impulsive pathways of risk for alcohol use disorder, Mol Psychiatry. (2015) 1-9

[8] G. Paxions, C. Watsons, The rat brain in stereotaxis coordinates, San Diego, CA Academic, 1986.

[9] R.M. Sears, H.C. Schiff, J.E. LeDoux, Molecular mechanisms of threat learning in the lateral nucleus of the amygdala, Prog Mol Biol Transl Sci. 122 (2014) 263-304.

[10] O.V. Severynovska., O.Ye. Pachomov, V.K. Rybalchenko, Etologija (osnovy povedinky tvaryn), Dnipropetr. Nat. Univ. Press, Dnipropetrovsk, 2010.

[11] F.Z. Shaw, R.F. Chen, H.W. Tsao, C.T. Yen, A multichannel system for recording and analysis of cortical field potentials in freely moving rats, J Neurosci Methods. 88(1) (1999) 33-43.

[12] H. Schweighöfer,C. Rummel, K Mayer, B. Rosengarten, Brain function in iNOS knock out or iNOS inhibited (l-NIL) mice under endotoxic shock, Intensive Care Med Exp. 2(1) (2014) 1-9.

[13] J. Wang, J. Wang, X. Li, D. Li, X.L. Li, J.S. Han, Y. Wan, Modulation of brain electroencephalography oscillations by electroacupuncture in a rat model of postincisionalpain, Evid Based Complement Alternat Med. (2013) 1-11.

[14] N.M. Zahr, Structural and microstructral imaging of the brain in alcohol use disorders. Handb Clin Neurol. 125 (2014) 275-290. 